Two observers in this series have been struck by the benefit obtained with penicillin in cases of infected socket. I have been impressed, in a series of 6 cases (seen outside the date range of the report), by the inefficacy of the drug. I found after some experimentation that $2 \frac{1}{2} \%$ red prontosil was much more effective. There are therefore some types of ocular inflammation which require much more extensive investigation before we can be sure how much to expect from the use of penicillin.

With regard to recurrence of inflammation I can say little yet. Because cases of one's own have not returned does not mean that there have been no recurrences; the patients may have gone to another part of the country. My opinion is that penicillin, being bacteriostatic and not bactericidal, will have little power to prevent recurrences unless it can be used, more or less as a routine, in intervals between exacerbations.

Finally, it must be remembered that many ocular inflammations are secondary to, or at least run parallel with, other affectionse.g., blepharitis and seborrhoeic dermatitis, conjunctivitis and rosacea, etc. The treatment of the underlying condition must not be lost sight of in the enthusiasm of employing a new method.

\section{Synopsis of Indications}

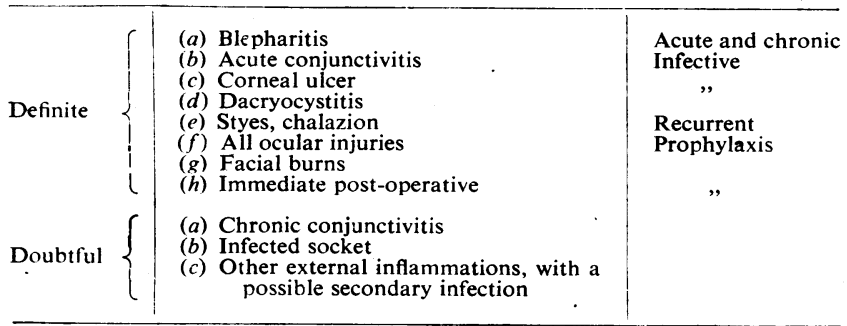

I believe penicillin to be quite useless in cases of deep keratitis, iritis, or cyclitis.

In conclusion I should like to emphasize the desirability of those concerned ensuring that all cases of facial burns with ocular involvement be given penicillin from the very beginning of treatment. In such a way I believe a number of ulcers from exposure keratitis can be avoided.

My thanks are due to Air Cdre. P. C. Livingston for making possible the supply of penicillin; to Squad. Ldrs. Sampson, Burr, and Palin for their co-operation, and for supplying me with their reports; to Squad. Ldr. R. J. O'Connor for the bacteriological culture work; to Squad. Ldr. W. I. Leslie for conducting experiments upon the time potency of penicillin; and to Sister M. C. E. Lodge for carrying out exact lines of treatment in my series of cases, thus ensuring uniformity of results.

\section{PENICILLIN AND SULPHONAMIDES IN INFANTILE GASTRO-ENTERITIS} BY

\section{ELIZABETH BURNS, M.B., B.Ch., D.C.H. AND}

W. GUNN, M.B., F.R.C.P. (North-Western Fever Hospital, L.C.C.)

The high incidence of acute gastro-enteritis in infants (familiarly known as D. and V.) continues to give cause for anxiety, showing a definite upward trend on the evidence of the figures of deaths from enteritis and diarrhoeal diseases in those under 2 years, which in England and Wales for 1939 to 1943 were : $2,812,2,891,2,985,3,415$, and 3,518 respectively. A disease predominantly of the hot months (hence the name summer diarrhoea) in the period before the first world war, it now shows no particular seasonal predilection except a tendency to increased severity and perhaps also increased prevalence during the period when catarrhal colds are rife, in the late winter and early spring months. In the absence of general notification (which should be made compulsory and be limited to the first year of life) estimates of the real incidence can only be conjectural, while mortality figures tend to be vitiated by inaccurate or incomplete certification, as exact diagnosis is not always easy in the absence of definite knowledge of the essential aetiology. Despite arguments to the contrary, there are no valid grounds for considering this disease to be different from so-called neo- natal diarrhoea, except for such differences as are attributable to the physiological characteristics of young babies. Workers in this field have recently inclined to the view that invasion of the upper respiratory tract, due most probably to a virus or group of viruses, is the primary cause, and the damage to the mucous membranes thereby sustained renders them more vulnerable to the ordinary bacterial flora of that region; whether the virulence of organisms harboured before attack may be enhanced in the process has not been definitely established. A somewhat similar process may eccur in respect of the normal intestinal bacteria, notably coliforms and enterococci; nor should it be forgotten that the same primary organism (causing identical disease processes so far as can be determined clinically) may gain entry by either the gastrointestinal or the upper respiratory tract, as has been proved in poliomyelitis.

The five recent cases described here, though representative, naturally belong to the severer form of the disease, which may range from the mildest grades, in which evidence of toxiinfective processes may be minimal or absent, to fulminating attacks. It is a matter of experience that the latter may sometimes respond to treatment more swiftly and completely than certain intermediate grades, the crucial difference apparently being the presence or absence of an infective focus and the success or failure of the measures employed to eradicate or neutralize it. All five cases were treated in single-bedded rooms (technically termed cells and often erroneously referred to as cubicles) in the same isolation unit and attended by the same medical and nursing staffs, with strict observance of aseptic nursing technique, including the wearing of "Cestra" type masks.

\section{Management of Cases}

To avoid unnecessary repetition, an outline of the management of the cases is given at this stage. A complete history of the patient from birth and the recent history of immediate contacts are followed by weighing of the patient and restoration measures such as warmth and fluids by mouth ; if dehydration is present an intravenous infusion of Hartmann's solution is given supplemented by $5 \%$ glucose at once or after a few hours, and by 4 parts solution to 1 part serum or plasma (the latter occasionally causes febrile reactions which may mislead) according to severity of symptoms and duration of disease, until metabolism is restored to normal as checked by level of plasma bicarbonate, proteins, urea, and chlorides, and by blood transfusion as shown by haematocrit readings and blood-count determinations. With experience these examinations may of ten be dispensed with, but avoidance of anaemia and oedema is best secured by periodic tests. The duration of the drip may vary from 1 to 5 days - average 3 days. Swabs of throat, nose, and nasopharynx (the most important site of the three) and specimens of faeces and urine are taken on admission and repeated as considered necessary. If vomiting persists only sips of Hartmann's solution or boiled water are given; but if it has ceased, increasing quantities are taken orally, deducting the amount from the drip intake. In 24 to 72 hours feeding is resumed, starting usually with a 1 in 4 dilution of a feed suitable for the age, rapidly reaching full strength according to appetite and tolerance : some infants can take only a 1 in 8 solution, while others can stand a 1 in 2 or even full-strength mixture from the outset, the remainder of fluid being given between feeds if the prescribed volume based on body weight is refused at the usual feeding-times. If there is no contraindication the food given before attack is chosen; if it was thought to disagree with the patient humanized national dried milk is the food of choice, and rarely requires modification such as citrated, peptonized, or acidified milk. Occasionally "Secway" may be needed to bridge the period between fluids and feeds. Difficulty at this stage is nearly always attributable to an infective focus, usually in the middle ear, occasionally in the renal pelves; and a sulphonamide, commonly sulphamezathine or sulphathiazole, is given either in the drip or orally with a view to preventing or even to securing resolution of the inflammatory process. Success is conditioned by the susceptibility of the causative organism, the natural resistance of the patient (specific immunity may likewise be a factor), and the nature and timing of such operative procedures as are adopted. Proper timing is considered of vital importance, as 
harm may be caused by premature operation as positively as by inadequate or tardy intervention.

The essential data, with critical comments, are given for each case in chronological order.

\section{Case I}

W. G.; 5/12 M., weight $15 \mathrm{lb} .4$ oz., artificially fed. May 21 , 1944: Onset-stools relaxed and green. Admitted 4th day; T. $100.6^{\circ} \mathrm{F}$. Moderate dehydration present; intravenous Hartmann's solution on following day with plasma later, and next day bilateral myringotomy, revealing sero-sanguineous exudate; bilateral cortical mastoidectomy 12 hours later; mucous membrane of the mastoid cells was inflamed and bone soft and red; no definite pus found. Wounds were stitched, leaving fine drainage-tube at lowest part for introduction of dilute penicillin ( 400 units per c.cm.) every 6 hours for 5 days; complete healing followed in 9 days. Throat and nasopharynx-Staph. aureus, $M$. catarrhalis, and coliforms; noseStaph aureus; right ear-M. catarrhalis, Staph. albus; left ear-no growth; mastoid bone-no growth.

Treatment.-Sulphathiazole $6 \mathrm{~g}$. in 4 days on admission, and sulphamezathine $12 \mathrm{~g}$. in 7 days, starting 5 days later. The patient did not improve-fever, diarrhoea, and loss of weight persisting despite four drips (three intravenous, one tibial marrow) and one blood transfusion (raising haemoglobin from 62 to $84 \%$ ). A trace of protein, some pus cells and red cells, and Staph. aureus and enterococci appeared in the urine at this stage (catheter specimen). Reopening the mastoids was considered unwarranted. Blood culture taken shortly before death on the 27th day of the disease revealed numerous Staph. aureus, partially sensitive to sulphonamides.

Necropsy.-Left mastoid-mucoid material; right-frank pus present, bone soft; from both, Staph. aureus and coliforms were isolated. Right middle cerebral vein thrombosed with purulent exudate along course.

Cominent.-Both myringotomy and mastoidectomy were probably undertaken too early; a more generous and longer course of sulphonamides advisable; mastoids might have been reopened with benefit. Coexisting renal damage may mislead, but appearance of pathogens in urine should suggest taking a blood culture.

\section{Case II}

B. W.; 6/12 M., weight $14 \mathrm{lb}$. $15 \mathrm{oz}$., artificially fed. May 24 , 1944 : Onset with fever, loose green stools, and bronchitis, for which sulphamezathine $9 \mathrm{~g}$. in 4 days was given. A week later vomiting and continued diarrhoea led to dehydration ; corrected by intravenous Hartmann's solution with glucose and plasma for 5 days and a second course of sulphamezathine $15 \mathrm{~g}$. in 5 days, of which $6 \mathrm{~g}$. was by the intravenous route; protein (a trace), scanty pus cells, and coliforms appeared in urine in the course of this treatment. Three weeks after onset bilateral myringotomy revealed muco-pus on both sides, containing Staph. aureus. No improvement followed, and cortical mastoidectomy was performed next day; the bone was softened and muco-pus was present in the mastoid cells, but cultures taken at operation proved negative. Fever continued, and blood culture taken 4 days after operation was found to grow penicillinsensitive Staph. aureus scantily. Penicillin 40,000 units every 24 hours was given by intravenous drip for 6 days and 5,000 units 3-hourly for an additional 2 days, when fever had just gradually subsided to normal. Blood culture was sterile on the day after starting penicillin therapy, which was continued to prevent relapse. As the penicillin-tube opening was still patent 13 days after operation and discharged mucopurulent material, reopening of the right mastoid was considered, but was deferred until rise of tèmperature or other sign of inflammation indicated; but subsequent recovery was rapid and uneventful.

Comment.-The negative bacteriological findings are possibly due to sulphonamide present in the swab material, para-aminobenzoic acid not being added to the culture media. The presence of renal complications did not seem to influence the course of the disease. Timely blood culture and use of penicillin saved the patient.

\section{Case III}

M. P.; 5/12 F., weight $11 \mathrm{lb}$. $11 \mathrm{oz}$., artificially fed. May 24 1944: Admitted with history of watery, green, offensive stools for 6 days, preceded by cough and nasal discharge; mild dehydration; T. $98.6^{\circ} \mathrm{F}$. The upper respiratory tract revealed nothing noteworthy apart from Str. viridans in the nasopharynx; urine normal. Some improvement followed a course of sulphamezathine, but 10 days later fever recurred with diarrhoea and pronounced dehydration, requiring an intravenous drip with plasma. Bilateral myringotomy 2 days afterwards yielded watery exudate containing Staph. albus and coliforms on the right side and blood only (sterile) on the left side. No improvement ensued, and a plasma drip followed by blood transfusion (haemoglobin raised from 75 to $95 \%$ ) was needed to sustain life. A second bilateral myringotomy gave a dry result, but mastoidectomy 4 days later revealed frank pus and necrosed bone (left) and soft readily bleeding bone (right), both sterile on culture. The blood was also sterile. With local penicillin healing of the wounds took place in 10 days. Some fever continued for 5 days after operation, but thereafter gain of weight and general progress were satisfactory.

Comment.-The negative bacteriological findings may have been due to sulphonamide either in the tissues killing the organism or on the swab inhibiting growth. Efficacy of mastoidectomy difficult to assess in such cases, although subsidence of fever is suggestive.

\section{Case IV}

P. L.; $8 / 12$ F., weight $11 \mathrm{lb} .11 \frac{1}{2} \mathrm{oz}$, artificially fed. June 10 , 1944: Onset sudden, with copious vomiting and loose, yellow, offensive stools; mcderate dehydration; temperature $99.6^{\circ} \mathrm{F}$. On admission bilateral myringotomy: left, sero-pus; right, blood only; both yielding Staph. albus. Upper respiratory tract revealed nothing of note apart from pneumococci in nasopharynx. Fever subsided for 3 days, when it recurred, necessitating an intravenous drip with plasma and 3 days later $100 \mathrm{c} . \mathrm{cm}$. blood, raising haemoglobin from 73 to $98 \%$. The urine was found to contain protein, numerous epithelial and pus cells, and scanty red cells; $B$. coli and proteus were found on culture. Blood culture was sterile. Fever and symptoms subsided in 3 days on a course of sulphathiazole, $10 \mathrm{~g}$. in 6 days, and remained settled. A month later the urine still contained $B$. coli on culture, and a trace of albumin; otherwise recovery appeared to be complete.

Comment.-Pyelitis seemed to be the sole cause of the gastroenteritis, responding rapidly to sulphonamide, but it may be merely a complication.

\section{Case $\mathbf{V}$}

R. B.; 2/12 M., weight $6 \mathrm{lb} .9$ oz., breast-fed for first 3 weeks. June 23, 1944: Cough and diarrhoea; general condition satisfactory. Admitted a week later with vomiting and frequent green, watery motions but without gross dehydration. Next day condition worse, requiring an intravenous drip. Throat and nose yielded Str. viridans. $M$. catarrhalis, and coliforms; nasopharynx-Staph. aureus and pneumococci; urine-trace protein, epithelial cells, $B$. coli, and proteus. Two days later myringotomy revealed a small amount of mucoid pus (right) and serous fluid (left), both containing Staph. aureus and albus, pneumococci, and coliforms. As high fever continued sulphamezathine $9 \mathrm{~g}$. in 4 days was given, and re-myringotomy was done 5 days later, again yielding small amounts of muco-pus and the same bacteria as before. Blood culture next day gave pneumococci Type 8 in uncountable numbers, sensitive completely to penicillin and partially to sulphonamide. Penicillin therapy, 5,000 units 3 -hourly intramuscularly, was s'arted, giving a level next day of $\pm 1-8$ after 3 hours, and the dosage was reduced to 4 -hourly intervals. The patient seemed better and took feeds readily, but 2 days later a generalized convulsion and meningitic signs appeared; lumbar puncture showed purulent fluid containing numerous pneumococci on culture. No response followed 3 injections of 5,000 units of penicillin given intrathecally at 8 -hour intervals, and the patient died 24 hours later.

Necropsy.-Inspissated pus in both middle ears and mastoid cells Greenish-yellow pus over entire surface of the brain, with subarachnoid haemorrhage over the base.

Comment-Sulphonamide was given too late in the disease to be effective; blood culture and lumbar puncture undertaken too late; penicillin probably sterilized the blood (a second culture was not taken, as the physical state was poor) but failed to prevent spread to the meninges, which very likely had taken place some hours or days before the convulsion which led to lumbar puncture.

\section{Discussion}

The cases recorded above illustrate well the diffculties encountered in locating the infective focus as well as in deciding the measures best calculated to eradicate it and the appropriate time to apply them. Although a history of cough or other symptom of respiratory infection was present in three cases the clinical evidence of such infection was negligible. While pyelitis occasionally appears to be solely responsible for gastro-enteritis, renal infection indistinguishable from mild pyelitis may occur at any stage of an attack, due primarily to upper respiratory infection, and should suggest the existence of bacteriaemia or septicaemia originating in mastoid suppuration. All three mastoidectomies were carried out under local anaesthesia, using $1 \%$ novocain preceded by $1 / 4$ gr. seconal. With careful infiltration pain is absent or minimal ; usually the patients suck their bottles contentedly during the operation. It seems warranted to conclude that, provided other possible causes are excluded, no patient suffering from gastro-enteritis should be permitted to die without operation ; if properly prepared for it with intravenous fluids, preferably including human serum or plasma, untoward effects are rare, and benefit some- 
times appears to accrue in cases in which evidence of mastoiditis was equivocal at the time of operation. The necessity for operation is governed by persistence of fever, diarrhoea, and/or vomiting and loss of weight despite every effort at resuscitation; the appearance under observation of enlarged posterior cervical glands gives support to the diagnosis, but neither their presence nor their absence should influence the final decision to operate.

There is general agreement that sulphonamides often fail to prevent the onset of mastoiditis and may actually be dangerous in masking the disease. Whether pericillin will succeed where sulphonamides have failed has not been adequately explored, but the present limited experience furnishes grounds for the hope that timely use of the drug will reduce appreciably the high case-fatality of infantile gastro-enteritis.

\section{Summary}

Five cases of severe acute gastro-enteritis in infancy, with two deaths, are described; in three patients mastoidectomy was performed, resulting in $(a)$ rapid resolution of suppurative processes found to be sterile at operation; (b) recovery from staphylococcal septicaemia cured by penicillin; $(c)$ death from staphylococcal septicaemia uninfluenced by sulphonamides. Of the remaining two cases one patient with $B$. coli pyelitis improved rapidly with sulphathiazole while the other died from pneumococcal meningitis and septicaemia, not responding to sulphonamide or penicillin therapy.

Our grateful thanks are due to Dr. R. Cruickshank, who carried out the bacteriological examinations, and to Miss Winifred Hall, who undertook the otological work.

\section{A SEVERE HAEMOLYTIC TRANSFUSION REACTION DUE TO THE Rh FACTOR BY}

A. BECK, M.D.Frankfurt, L.R.C.P.\&S.

Pathologist, Public Health Laboratory, Burnley

C. V. HARRISON, M.D.

Department of Pathology, Liverpool University AND

J. M. OWEN, M.B., Ch.B., D.R.C.o.G.

R.M.O., Bank Hall Maternity Hospital, Burnley

The demonstration of the $\mathrm{Rh}$ factor in human blood cells (Landsteiner and Wiener, 1940) has led to the elucidation of many previously unexplained haemolytic reactions following the transfusion of apparently compatible blood. Later workers (Levine et al., 1941 ; Boorman et al., 1942 ; Race et al., 1943) have shown that the antibody responsible for the haemolysis may develop in an Rh-negative person either as a result of previous transfusions with Rh-positive blood or, in the case of women, as a result of bearing an Rh-positive foetus. In the latter case the infant usually suffers from haemolytic disease of the newborn. In the present case a severe haemolytic reaction followed the transfusion of $\mathrm{Rh}$-positive blood in a woman who had previously been transfused (probably with Rh-positive blood) and also had borne several Rh-positive children. It is not known which of these two factors was responsible for the production of the antibody.

\section{Case Record}

Mrs. A., aged 38, had a history as follows:-1929: Forceps delivery; normal baby, Group $\mathrm{A}_{2}$ Rh-positive. 1932: Normal delivery, but retained placenta ; baby healthy at birth but died at 5 months; cause unknown. 1936: Normal delivery followed by postpartum haemorrhage. Was given blood transfusion (group not known). Baby healthy; Group O Rh-negative. 1940: Normal delivery. This child, who is Group $A_{2}$ Rh-positive, was said to have been jaundiced for four weeks after birth. 1942: Twins. First infant stillborn owing to hydrocephalus; second infant healthy, Group $A_{2}$ Rh-negative. Labour followed by severe haemorrhage. Two transfusions of Group A blood were given, with an interval of 14 days; both were followed by severe rigors.

In 1944 she was seen when 22 weeks pregnant. Urine and blood pressure were normal. In view of a moderate anaemia $(\mathrm{Hb} 55 \%$, R.B.C. $3,600,000$ ) it was decided to give her a blood transfusion. One pint of Group A blood was administered. One hour later she started to vomit, her pulse became weak, and she complained of lumbar pain. Anuria set in and alkali therapy was begun immediately. The following morning she was jaundiced. The daily urinary output for the next six days varied between 1 and $4 \mathrm{oz}$. The urine was dark red in colour and was loaded with albumin. On the fifth day she complained of severe headache, and the next day some oedema of the legs was noticed. On the eighth day normal diuresis set in and her condition gradually improved. On the thirteenth day she left hospital against advice, but had apparently recovered from her haemolytic reaction. The following day she returned with an incomplete abortion. The placenta had to be removed under general anaesthesia. She collapsed before the operation, but rallied sufficiently for it to be completed. She died four hours later, apparently from shock.

A necropsy was performed 24 hours after death. Apart from signs of shock, the significant findings were those related to the haemolytic transfusion reaction. The liver showed signs of fatty change. The kidneys were enlarged to twice their normal size and showed a pale-grey colour with an indistinct cortical pattern. The spleen was greatly enlarged $(580 \mathrm{~g}$.) and firm. The abdominal lymph glands were also enlarged.

Microscopical examination of the kidneys revealed the characteristic lesions of "transfusion kidney" as described by previous workers (De Gowin et al., 1938; De Navasquez, 1940). The organ differed from that in average cases in having numerous focal collections of lymphocytes, macrophages, and eosinophils in both cortex and medulla. Similar changes have, however, been described by Goldring and Graef (1936) and by Ayer and Gauld (1942). The liver showed fatty change and slight degeneration towards the centres of the lobules. The spleen, in addition to siderosis, showed marked proliferation of the histiocytic cells of both the pulp and the Malpighian bodies. The abdominal lymph glands presented a similar picture.

The patient's blood was examined on the 2 nd and 12th days after transfusion. The donor was Group A Rh-positive. The patient was Group A Rh-negative, and on the first examination, two days after transfusion, no agglutinable Rh-positive cells were found, indicating that all the transfused cells had been destroyed. On this occasion Rh antibody was demonstrable in the undiluted serum. On the 12th day the $\mathrm{Rh}$ antibody titre had risen from $1 / 1$ to $1 / 8$. The husband was found to be Group $A_{2}$ Rh-positive ; the blood groups of the children have already been given.

An interesting problem in this case is which of the various possible factors was responsible for the production of $\mathrm{Rh}$ antibody. Of the patient's four surviving children two are known to be Rh-positive. It is stated that one of these-the third-was jaundiced for four weeks after birth, and it is probable that this was due to haemolytic disease of the newborn; the absence of signs of haemolytic disease in the case of the fourth child would be due to its being Rh-negative. It is therefore at least possible that the production of $\mathrm{Rh}$ antibody may have been due to repeated pregnancies. The $R h$ group of the blood given in the earlier three transfusions is not known, but on probabilities it is likely that most, if not all, were $R h$-positive. Also, the fact that the second and third were followed by reactions is suggestive. The other interesting feature is the remarkable size and reaction of the spleen. There was no evidence of leukaemia in a blood count done shortly before the last transfusion, and neither the necropsy findings nor the histology indicate reticulosis.

We believe that the reticulo-endothelial hyperplasia in the spleen was the result of stimulation by incompatible transfusions; probably two transfusions in 1942 and certainly one a fortnight before death.

\section{Summary}

A case is described of a severe haemolytic reaction following the transfusion of $\mathrm{Rh}$-positive blood into an $\mathrm{Rh}$-negative woman who had borne two Rh-positive children and had previously had three transfusions. It is not known which of these two possible factors gave rise to the $\mathrm{Rh}$ antibody.

\section{REFERENCES}

Ayer, G. D., and Gauld, A. G. (1942), Arch. Path, 33, 513

Boorman, K.' E.. Dodd, B. E., and Mollison, P. L. (1942). British Medical Journal, $2,535,569$

De Gowin, E. L., Warner, E. D., and Randall, W. L. (1938). Arch. intern. Med., $61,609$.

De Navasquez, S. (1940). J. Path. Bact., 51, 413.

Goldring, W., and Graef, I. (1936). Arch. intern. Med., 58, 825.

Landsteiner, K., an 1 Wiener, A. S. (1940). Proc. Soc. exp. Biol., N.Y. 43, 223. Levine, P., Burnham L., Katzin, E. M., and Vogel, P. (1941). Amer. J. Obstet. Gynec., 42, 925

ace, R. R., Taylor, G. L., Cappell, D. F., and McFarlane, M. N. (1943). British Medical Journal, 2, 289. 